\title{
Simulation Test of Sensor Response Speed of EMG Signal Based on Finite Element Analysis
}

\author{
Nai Wang \\ Department of Physical Education, Henan College of Traditional Chinese Medicine, Zhengzhou, \\ 450046, China \\ wang_nai2014@yeah.net
} Keywords: Electrical tomography, Finite element, Reaction rate, GPU processor, VC++,
Reconstruction.

\begin{abstract}
In order to test the attention distribution training on the effect of Sanshou athletes training, this paper presents a new signal acquisition and processing technology in the process of action reaction speed and attack accuracy analysis, the technology uses electrical tomography technology (ET) to carry on sensor arrangement for the muscle EMG signal, and the obtained image carries out the finite element reconstruction. In order to meet the demand of large data calculation, using GPU as a computing processor optimizes the computing model using clustering algorithm, and using $\mathrm{VC}++$ software carries out programming design of the algorithm. Through the image reconstruction of Sanshou technology action, we can get the significant analysis results of attention distribution training on Sanshou technology action reaction speed and attack accuracy, which provides a new computer method for the Sanshou athletes training.
\end{abstract}

\section{Introduction}

In all sports, Sanshou has distinct characteristics, it mainly displays in quick reaction and attack with high accuracy [1]. Sanshou is a competitive sport, it must be completed by two people together, and it is formally started in the game, Sanshou athletes must be responsive and accurate action to get points in the fight against [2,3]. In order to ensure the Sanshou competition rules to further perfect, we modify the Sanshou competition rules, the new rules adds punches and leg score, reducing the takedown action score. After the implementation of game new rules, we further improve the response speed and authentic requirements of Sanshou athletes [4-6]. This study uses the ET image reconstruction technique and cluster analysis algorithm to analyze the Sanshou movement image, its basic frame is shown in Figure 1.

ET

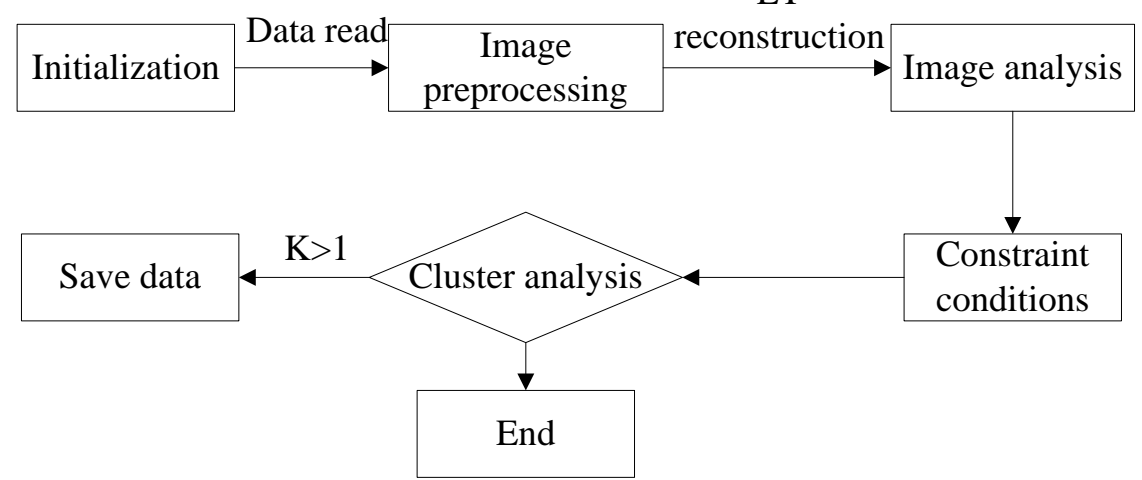

Fig.1: The basic framework of action ET image reconstruction analysis

Figure 1 indicates the basic framework map of the Sanshou movement analyzed by ET image reconstruction technique. The basic process carries out the signal acquisition on the Sanshou movement image, the obtained image carries out the pre-processing, and then using ET reconstruction technique carries out image analysis to obtain the constraint conditions of image analysis, at the same time using the cluster analysis clusters the constraint condition data [7-9]. Finally through the calculation, we can get the computing data of Sanshou athletes' reaction speed and attack accuracy. 


\section{Design of Actions Image ET Reconstructed Cluster Mathematical Model and Algorithm}

When the human body muscle contracted, placing the electrodes in the skin surface will collect the corresponding muscle action potential, i.e. the surface EMG signals [10,11]. The use of ET reconstruction technology can realize the image reconstruction processing of electromechanical signal, this study uses clustering algorithm, in which clustering function is shown in Formula (1).

$$
Y\left(x_{j}\right)=0.02+\sum_{i=1}^{n} \frac{1}{i+\sum_{j=1}^{n}\left(x_{j}-a_{j i}\right)^{n}} .
$$

Among them, $a$ represents judgment coefficient matrix, $x$ represents image reconstruction function. Assuming the samples of ET image reconstruction is $b_{k}=\left(b_{k 1}, b_{k 2}, \cdots b_{k n}\right)$, then the clustering distance of $b_{k}$ and $b_{l}$ is shown in formula (2).

$$
\rho\left(b_{k}, b_{l}\right)=\sqrt{\sum_{z=1}^{n}\left(b_{k z}-b_{l z}\right)}, k, l=1,2, \cdots n \text {. }
$$

The density of data sample point ${ }^{b_{k}}$ can be expressed as

$$
M_{k}=\sum_{l=1}^{n} \frac{\rho\left(b_{k}, b_{l}\right)}{\sum_{k=1}^{n} \rho\left(b_{k}, b_{l}\right)}, k, l=1,2, \cdots n
$$

So we can get the center distance of the ET image reconstruction, the specific expression is shown in formula (4).

$$
M p_{k}=\exp \left(-\frac{M_{k}}{\sum_{i=1}^{n} \rho\left(b_{l}, b_{k}\right)}\right)
$$

According to the mathematical model of clustering algorithm, we can use the VC++ program to carry on the algorithm programming, in which the main algorithms are as follows [12-14]:

double* a=new double[col]; double* b=new double[col];

double**dis $=$ new double $^{*}[\mathrm{k}]$;

$$
\text { for }(\mathrm{i}=0 ; \mathrm{i}<\mathrm{k} ; \mathrm{i}++)
$$

$\{$ dis[i]=new double[row $] ;\}$

for $(\mathrm{i}=0 ; \mathrm{i}<\mathrm{k} ; \mathrm{i}++)$

$\{$ for $(\mathrm{t}=0 ; \mathrm{t}<\mathrm{col} ; \mathrm{t}++)$

$\{\mathrm{a}[\mathrm{t}]=$ center $[\mathrm{i}][\mathrm{t}] ; \quad\}$

for $(j=0 ; j<$ row; $j++)$

$\{$ for $(\mathrm{t}=0 ; \mathrm{t}<\mathrm{col} ; \mathrm{t}++)$

double $\mathrm{d}=0$;

$\{\mathrm{b}[\mathrm{t}]=\operatorname{data}[\mathrm{j}][\mathrm{t}] ;\}$

$$
\begin{aligned}
& \text { for }(\mathrm{t}=0 ; \mathrm{t}<\mathrm{col} ; \mathrm{t}++) \\
& \left.\begin{array}{l}
\{\mathrm{d}+=(\mathrm{a}[\mathrm{t}]-\mathrm{b}[\mathrm{t}]) *(\mathrm{a}[\mathrm{t}]-\mathrm{b}[\mathrm{t}]) ; \quad\} \\
\operatorname{dis}[\mathrm{i}][\mathrm{j}]=\operatorname{sqrt}(\mathrm{d}) ; \quad\}\}
\end{array}\right\}
\end{aligned}
$$

\section{Analysis of the Sanshou Movement based on the ET Image Clustering Reconstruction}

In order to verify the effectiveness and reliability of image clustering reconstruction mathematics model and algorithm designed in second part, this paper uses the ET tomography technology to study the reaction rate of Sanshou muscle action, in which the layout of sensor strain gauge is as shown in Figure 2. 


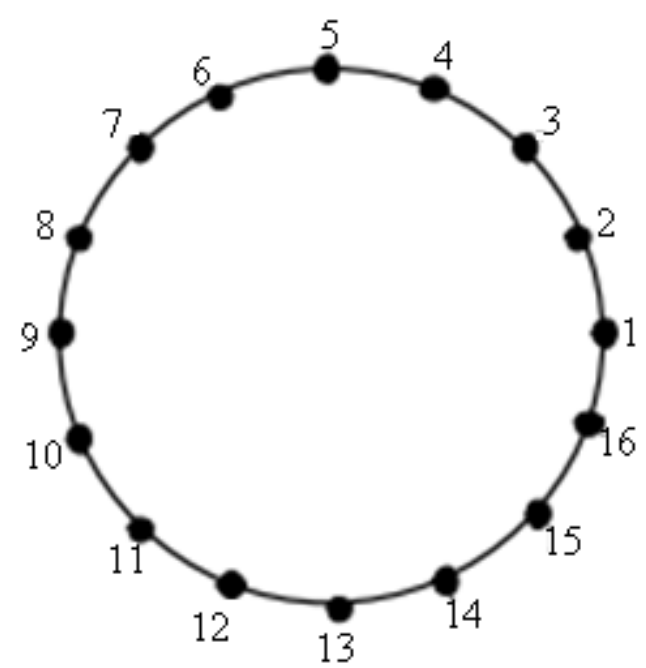

Fig.2: Schematic diagram of the ET sensor layout

Electrical tomography can be summarized as the placement of $\mathrm{N}$ electrodes in a peripheral section boundary spacing of the muscle, it turns to the measuring electrode to apply an excitation signal, and this process is established sensitive field inside the object to measure the output signal between other electrode.

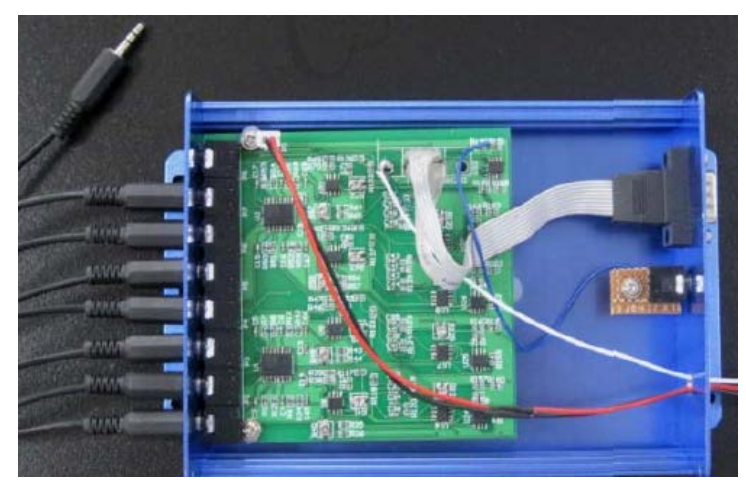

Fig.3: EMG signal acquisition physical map

As shown in Figure 3, it represents the physical map of the EMG signal acquisition. Sanshou movement attack speed and attack accuracy mainly use EMG signal acquisition to complete, in which wires are mutually vertical, oblique or bend line in the circuit wiring to avoid mutual parallel, reducing the parasitic coupling.

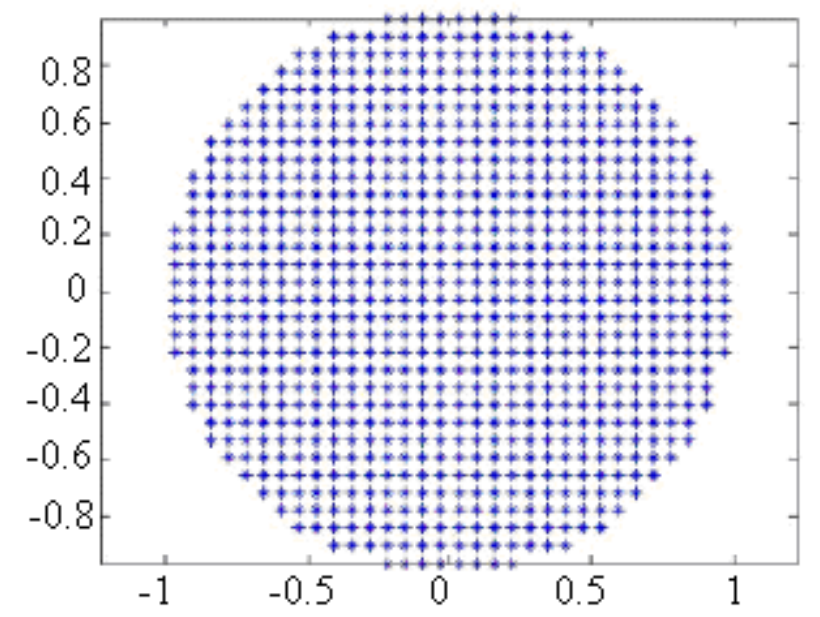

Fig.4: ET image 3D reconstruction

In Figure 4, the use of finite element method can complete the 3D model reconstruction of ET images. Due to the large amount of 3D model calculation, it has higher requirements on computing speed and storage capacity, this study uses GPU processor as the calculation core device [15-17]. By clustering calculation, the results can obtained as shown in Figure 5. 


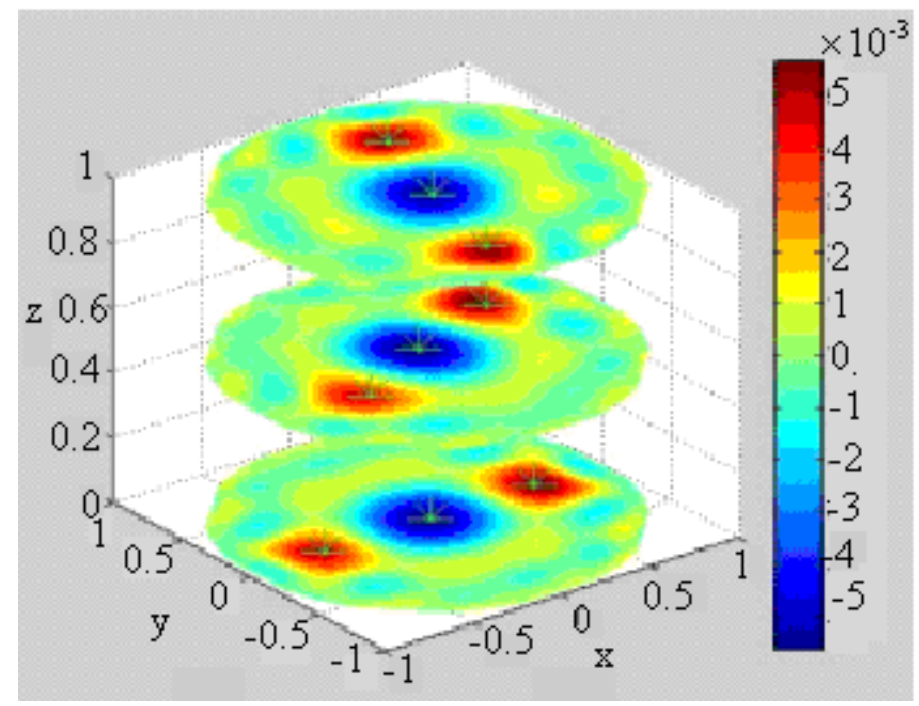

Fig.5: ET 3D image clustering center

As shown in Figure 5, the use of clustering algorithm can effectively get the clustering center of three-dimensional images. After using ET tomography technology, we can get accurate clustering center, and the clustering center position is also more accurate, in which the reaction time with the changes of the training times is shown in Figure 6.

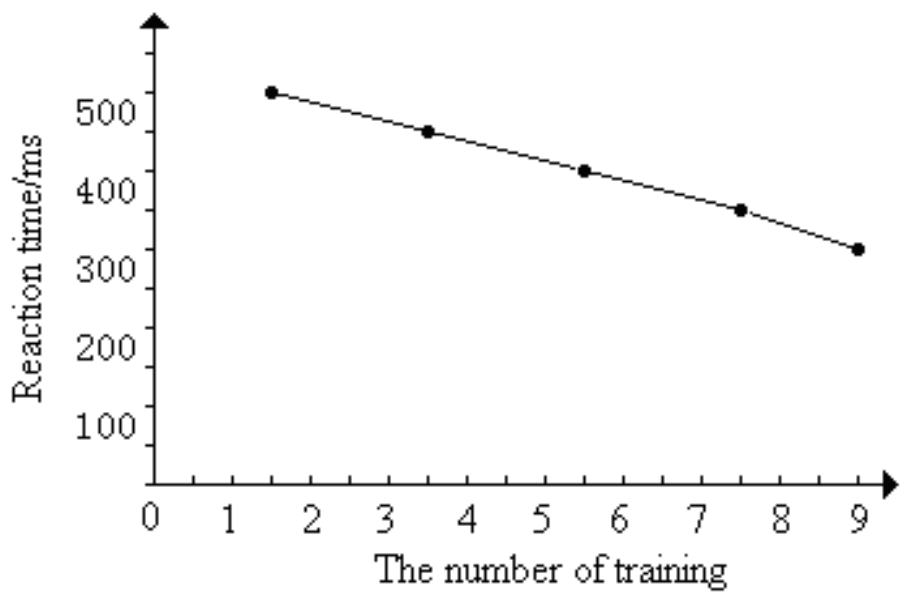

Fig.6: The reaction time with the changes of the training times

This study is the selection of 10 subjects to carry on the simple reaction time test of the 9 times. Through the ET image reconstruction technology, we draw the reaction time curve with the changes of the training times, it can be seen from the results that with the increase of training times, reaction time becomes smaller gradually, this shows that distribution training has a significant impact on the response time of the athletes $[18,19]$. In order to further study the effect of significantly, the experimental group and the control group carry out comparison, the results are shown in Table 1.

Table 1: The image processing results of Sanshou athletes' reaction speed and action accuracy

\begin{tabular}{llllll}
\hline & & Average value & Variance & $\mathrm{F}$ & $\mathrm{P}$ \\
\hline \multirow{2}{*}{ Reaction time } & Experimental group & 278.86 & 55.26 & 0.13 & 0.78 \\
& Control group & 296.25 & 38.21 & & \\
\multirow{2}{*}{ Accuracy rate } & Experimental group & 0.78 & 0.56 & \multirow{2}{*}{1.0} & 0.33 \\
& Control group & 0.56 & 0.48 & &
\end{tabular}

As shown in Table 1, the action characteristics of Sanshou players carry out 3D image processing on the attention distribution training, we have obtained Sanshou athletes' reaction speed and action accuracy after training. Sanshou athlete's response action and speed accuracy after attention distribution training carries out single factor variance analysis, the training results show that whether it is the reaction time and accuracy, it goes through the attention distribution training, the 
results are better than the control group, it illustrates the attention distribution plays an obvious effect on the training of athletes.

\section{Summary}

(1) Based on the accurate analysis method of motion images, this paper proposes a new image reconstruction algorithm, the algorithm uses the ET reconstruction technique as the basic frame of image reconstruction, and the use of clustering algorithm optimizes the design of reconstruction model.

(2) In order to satisfy the high capacity and large data calculation, this paper uses GPU as the core calculation processor, and using ET reconstruction technique carries out layout on the sensor. Through the EMG signal processing obtained by acquisition, we can get the relevant data of the reaction speed and attack accuracy in Sanshou technology action, which provides a theoretical reference for the analysis of Sanshou movement.

\section{References}

[1] S.Y. Ma. Analysis of Taekwondo starting operation. Contemporary sports science and technology, 2012(3): 45-48.

[2] Y.F. Li, J. Cao. Study of the response levels of Adolescent tennis athletes. Hubei sports science and technology, 2011(6): 91-95.

[3] X.P. Chen. Study of affecting the reaction rate factors and the training method. Sports scientific literature review, 2011(3): 12-16.

[4] Y.P. Li, Q.J. Pang. Study of the effects of the athletes' reaction velocity factor. Science and technology for development, 2011(2): 45-48.

[5] J. Miao, X.D. Zhang, F. Yu. Discussion on the Sanshou coaches on the development of athletic psychological ability. Fight, 2011(1): 23-26.

[6] D.L. Zhang. Comparative study of Sprinters' start reaction time at the twenty-ninth Olympic Games. Journal of Zhaoqing University, 2012(5): 78-80.

[7] Q. Fu. Characteristics study of high level fencing visual reaction time. Journal of Capital Institute of Physical Education, 2012(5): 34-37.

[8] Q. Zhang, Y.G. Sun, T. Yang et al. Application of wireless sensor network in intelligent power grid. Chinese electric power, 2011, 43(6): 31-36.

[9] L.J. Chen, Y.C. Mao, D.X. Chen et al. Study of the average constraint wireless sensor network topology control. Chinese Journal of computers, 2011, 30(9): 1544-1550.

[10] W.F. Cheng, X.K. Liao, C.X. Shen. Study of the maximum coverage scheduling algorithm of directional sensor networks. Journal of software, 2011(4): 975-984.

[11] J. Wen, J. Jiang, W.H. Dou. Fair directional sensor network direction optimization and node scheduling algorithm. Journal of software, 2011, 20(3): 644-659.

[12] D. Tao, H.D. Ma, L. Liu. Study of the path coverage enhancement algorithm in video sensor network. Chinese Journal of electronics, 2012, 36(7): 26-28.

[13] Q.P. Sun, L. Gao. Study of our country excellent men Sanshou athletes' sport cognitive trait anxiety. Shandong sports science and technology, 2012, 3(30): 45-48.

[14] Y.G. Ceng. Study of Sanshou athletes' reaction speed and method. Tsinghua Science and technology, 2012, 4(1): 24-27.

[15] Y. Chang. Comparative study of adolescent Sanshou athletes and adult Sanshou athletes reaction. Fight (Wushu science), 2012, 3(4): 56-59.

[16] N. Fu, L.Y. Qiao, X.Y. Peng. The blind estimation algorithm of sparse source mixed matrix based on improved K-means clustering and Hof transform. Journal of electronics, 2012, 37(4A): 92-96

[17] Y. Song, A.A. Liu, Y.D. Zhang. Research on video letter extraction method based on clustering. Journal of China Institute of communications, 2011,30 (2): 136-140. 
[18] Y.X. Lu. Research on the mobile communication enterprise customer segmentation method. Dalian University of Technology, 2011(19): 81-84.

[19] T. Zhou. Rough K-means clustering algorithm with adaptive parameters. Engineering and computer application, 2010, 46 (26): 7-10. 